\title{
Serological responses to COVID-19 booster vaccine in England
}

Georgina Ireland, ${ }^{1 *}$ Heather Whitaker, ${ }^{2 *}$ Shamez N Ladhani, ${ }^{1,7}$ Frances Baawuah, ${ }^{1,3}$ Vani Subbarao, ${ }^{1}$

Ezra Linley, ${ }^{4}$ Lenesha Warrener, ${ }^{5}$ Michelle O'Brien, ${ }^{3}$ Corinne Whillock, ${ }^{1}$ Paul Moss, ${ }^{6}$ Mary E

Ramsay ${ }^{1}$, Gayatri Amirthalingam ${ }^{* *},{ }^{1}$ Kevin E Brown**,

* These authors contributed equally

** These authors jointly supervised this work

1. Immunisation and Vaccine Preventable Diseases Division, UK Health Security Agency ${ }^{\$}$, London, United Kingdom

2. Statistics, Modelling and Economics Department, UK Health Security Agency $\$$, London, United Kingdom

3. Brondesbury Medical Centre, Kilburn, London, United Kingdom

4. Sero-Epidemiology Unit, UK Health Security Agency $\$$, Manchester, United Kingdom

5. Virus Reference Department, UK Health Security Agency ${ }^{\$}$, London, United Kingdom

6. Institute of Immunology and Immunotherapy, University of Birmingham, Edgbaston, United Kingdom

7. Paediatric Infectious Diseases Research Group, St. George's University of London, London, United Kingdom

\$ From Friday 1st October 2021, the UK Health Security Agency (UKHSA) became fully operational. UKHSA takes on the health protection responsibilities of Public Health England (PHE) and incorporates NHS Test \& Trace and the Joint Biosecurity Centre (JBC). UKHSA is an executive agency of the Department of Health and Social Care. It is responsible for planning, preventing and responding to public health threats, and providing intellectual, scientific and operational leadership at national and local level, as well as on the global stage.

Correspondence: Georgina Ireland (Georgina.ireland@phe.gov.uk)

Keywords: COVID-19; COVID-Vaccine; Antibody; Spike Protein; Immunity; Pfizer; AstraZeneca 


\begin{abstract}
Introduction: There are limited data on immune responses after COVID-19 vaccine boosters in individuals receiving primary immunisation with BNT162b2 (Pfizer-BioNTech) or AZD1222 (AstraZeneca).
\end{abstract}

Methods: A prospective, cohort study to assess SARS-CoV-2 antibody responses before and after booster vaccination with BNT162b2 in adults receiving either (i) two BNT162b2 doses $<30$ days apart (BNT162b2-control), (ii) two BNT162b2 doses $\geq 30$ days apart (BNT162b2-extended) or (iii) two AZD1222 doses $\geq 30$ days apart (AZD1222-extended) in London, England. SARS-CoV-2 spike protein antibody geometric mean titres (GMTs) before and 2-4 weeks after booster were compared.

Results: Of 750 participants, 626 provided serum samples for up to 38 weeks after their second vaccine dose. Antibody GMTs peaked at 2-4 weeks after the second dose, before declining by $68 \%$ at 36-38 weeks after dose 2 for BNT162b2-control participants, $85 \%$ at 24-29 weeks for BNT162b2extended participants and 78\% at 24-29 weeks for AZD1222-extended participants. Antibody GMTs was highest in BNT162b2-extended participants (942 [95\%Cl, 797-1113]) than AZD1222-extended (183 [124-268]) participants at 24-29 weeks or BNT162b2-control participants at 36-38 weeks (208; $95 \% \mathrm{Cl}, 150-289)$. At 2-4 weeks after booster, GMTs were significantly higher than after primary vaccination in all three groups: $18,104(95 \% \mathrm{Cl}, 13,911-23,560 ; n=47)$ in BNT162b2-control (76.3fold), 13,980 (11,902-16,421; $\mathrm{n}=118)$ in BNT162b2-extended (15.9-fold) and 10,799 (8,510-13,704; $\mathrm{n}=43$ ) in AZD1222-extended (57.2-fold) participants. BNT162b2-control participants (median:262 days) had a longer interval between primary and booster doses than BNT162b2-extended or AZD1222-extended (both median:186 days) participants.

Conclusions: We observed rapid serological responses to boosting with BNT162b2, irrespective of vaccine type or schedule used for primary immunisation, with higher post-booster responses with longer interval between primary immunisation and boosting. Boosters will not only provide additional protection for those at highest risk of severe COVID-19 but also prevent infection and, therefore, interrupt transmission, thereby reducing infections rates in the population. Ongoing surveillance will be important for monitoring the duration of protection after the booster. 


\section{Introduction}

Two doses of BNT162b2 (Pfizer-BioNTech/Comirnaty ${ }^{\circledR}$ ) or AZD1222 (AstraZeneca/Vaxzevria ${ }^{\circledR}$ ) vaccines provide high levels of protection from COVID-19, hospitalisation and death for at least 4-6 months after vaccination. ${ }^{1}$ Unlike other countries that that offered COVID-19 vaccination according to the authorised 3-4 week interval, the UK recommended an extended 12-week schedule to expedite the rollout of the first dose of vaccine. ${ }^{1}$ Subsequent studies have demonstrated higher antibody levels after the second dose with the extended schedule than the authorised interval, potentially providing better longer-term protection. ${ }^{1,2}$ However, antibody levels and clinical protection wane over time. In England, vaccine effectiveness (VE) against hospitalisation declined to $77.0 \%$ and $92.7 \%$ beyond 20 weeks post-vaccination and $78.7 \%$ and $90.4 \%$ against death for AZD1222 and BNT162b2, respectively, with greater waning among older adults and those with underlying comorbidities. ${ }^{2}$ As these groups were the first to be offered COVID-19 vaccination and because of concerns about waning immunity, emergence of the highly-transmissible Delta variant which causes more severe disease and can infect vaccinated individuals, ${ }^{3,4}$ high and sustained community infection rates in the UK, and winter pressures on the national healthcare system, the UK Joint Committee on Vaccination and Immunisation (JCVI) recommended a third dose (booster) with either a single dose of BNT162b2 or a half dose $(50 \mu \mathrm{g})$ of mRNA-1273 (Moderna/Spikevax ${ }^{\oplus}$ ) for adults aged $\geq 50$ years, individuals aged $16-49$ years in clinical risk groups, adult carers and household contacts of immunosuppressed individuals, and frontline health and social care workers, to be offered at least 6 months after their second dose. ${ }^{5-7}$

In England, the UK Health Security Agency (UKHSA) initiated an evaluation of vaccine responses in adults aged $\geq 50$ years who received the BNT162 b2 or AZD1222 as part of the national immunisation programme to compare short versus longer interval vaccine schedules and monitor antibody waning over time. ${ }^{8}$ Here, we describe the antibody kinetics after primary immunisation and booster vaccination in adults aged $\geq 50$ years who were vaccinated as of the national COVID-19 immunisation programme.

\section{Methods}

The COVID-19 vaccine responses after extended immunisation schedules (CONSENUS) cohort has been described previously. ${ }^{1,8}$ CONSENSUS recruited immunocompetent adults aged $\geq 50$ years in January 2021 in London to provide serial blood samples at 0,3,6,9,12,15 and 20 weeks after their first dose of COVID-19 vaccine. As part of the national COVID-19 vaccine roll out, participants received either (i) two BNT162b2 doses <30 days apart (BNT162b2-control), (ii) two BNT162b2 doses 
$\geq 30$ days apart (BNT162b2-extended) or (iii) two AZD1222 doses $\geq 30$ days apart (AZD1222extended). Additional blood samples were taken before and after the booster.

Serum samples were tested for nucleoprotein $(\mathrm{N})$ antibodies as a marker of previous SARS-CoV-2 infection (Elecsys Anti-SARS-CoV-2 total antibody assay, Roche Diagnostics, Basel, Switzerland: positive $\geq 1 \mathrm{COI}$ ) and spike (S) protein antibodies, which could be infection- or vaccine-derived (Elecsys Anti-SARS-CoV-2 S total antibody assay, Roche Diagnostics: positive?? $\geq 0.8$ arbitrary units $(\mathrm{au}) / \mathrm{mL}$ to assess vaccine response). Antibody geometric mean titres (GMTs) were calculated with 95\% confidence intervals ( $\mathrm{Cl}$ ). Geometric mean ratios (GMR) of responses between timepoints were estimated using a mixed regression model on log responses including a random effect for each participant; separate models were fitted for each vaccine group. The GMR of responses by vaccine type at each post-vaccination timepoint was estimated via regression on $\log$ Roche $S$ responses and included age-group and sex. Statistical analyses were performed using STATA v.14.2. Individuals with $\geq 0.4(\mathrm{au}) / \mathrm{mL}$ on the Roche $\mathrm{N}$ assay were considered to have had prior SARS-CoV-2 infection.

\section{Results}

\section{Antibody kinetics following primary course}

Of the 750 recruited participants, 626 provided serum samples for up to 38 weeks after their second dose of vaccine (Table 1). Antibody GMTs peaked at 2-4 weeks after the second dose and then declined for all three vaccine schedule groups for all subsequent sampling points (Table 2). Antibody GMTs declined by $68 \%$ at $36-38$ weeks after dose 2 for BNT162 b2-control participants, $85 \%$ at $24-29$ weeks for BNT162b2-extended participants and 78\% at 24-29 weeks for AZD1222-extended participants. Antibody GMTs at 24-29 weeks remained higher in the BNT162b2-extended group than in AZD1222-extended participants ( 942 [95\%Cl, 797-1113] vs 183 [124-268]; $p<0.001$ ), while the latter was similar to BNT162b2-control participants at 36-38 weeks (208 [150-289]).

Regardless of primary vaccination type or schedule, antibody GMTs at all timepoints after dose 2 were greater in previously-infected participants. In AZD1222-extended participants, however, the decline in GMTs was smaller at 18-23 weeks in previously-infected than in uninfected participants (49\% vs $68 \%$ ). By comparison, a similar decline was observed for previously-infected compared to uninfected BNT162b2-extended participants at $24-29$ weeks ( $80 \%$ vs $85 \%$ ).

\section{Post-booster response}

Serum samples were available for 52 BNT162b2-control, 131 BNT162b2-extended and 50 AZD1222extended participants 2-4 weeks after the BNT162b2 booster. The boosted AZD1222-extended participants had a shorter interval between primary doses than all AZD1222-extended participants in 
the evaluation ( 51 days vs 72 days), while BNT162b2-control participants (median:262 days) had a longer interval between primary and booster doses than BNT162b2-extended or AZD1222-extended participants (both median:186 days).

Antibody GMTs at 2-4 weeks were highest in BNT162b2-control $(18,104,95 \% \mathrm{Cl}$ : 13,9113-23,560), followed by BNT162b2-extended (13,980, 95\%Cl: 11,902-16,421) and AZD1222-extended (10,799, 95\%Cl: 8,510-13,704) (Table 2). GMTs in BNT162 b2-control were greater than for AZD1222extended participants $(p=0.01)$. The largest post-booster increase in GMTs was in BNT162b2-control participants (76.3-fold), followed by AZD1222-extended (57.2-fold) and BNT162b2-extended (15.9fold) (Table 2). Booster responses were not affected by age (all $p<0.05$ ) but higher in females $(p=0.008)$. Sufficient post-booster information was available for previously-infected AZD1222extended and BNT162b2-extended participants, where geometric mean responses increased 9.9fold (to $45,430,95 \% \mathrm{Cl}$ : $30,374-67,947$ ) and 4.9-fold (to $18,604,95 \% \mathrm{Cl}: 12,899-26,832$ ) respectively.

\section{Discussion}

These early data show a rapid serological response to boosting with BNT162b2, with significantly higher antibody responses than after the second dose. Importantly, our cohort consists primarily of older adults who have a higher risk of severe COVID-19 and are, therefore, most likely to benefit from vaccination. Early data from Israel and England demonstrate significantly better protection against severe COVID-19, hospitalisation and death after booster vaccination. ${ }^{2,9}$ In England, 14 days after boosting with BNT162b2 among $\geq 50$ year-olds, VE was similar in individuals who had received only 2 primary doses of BNT162b2 (87.4, 95\% 8 Cl $89-89.4)$ and AZD1222 $(84.4,95 \% \mathrm{Cl} 82.8-85.8)$ at least 140 days previously, although the analysis did not separate by schedule. ${ }^{2}$ The higher post-booster GMTs in BNT162b2-control participants, who were the first to be vaccinated, is likely due to the extended interval between primary and booster vaccines, allowing more time for enhancing immune memory and greater waning of antibodies, both of which are likely to enhance post-booster responses.

In conclusion, we observed very high antibody responses following a BNT162b2 booster, irrespective of vaccine or schedule used for primary immunisation. A longer interval between primary immunisation and the booster provides higher post-booster antibody responses and, potentially, longer lasting protection. Decisions on timing of booster doses should take account of the current and predicted epidemiological context to ensure the most vulnerable groups are optimally protected during heightened periods of transmission. Whilst the rates of severe disease, hospitalisations and deaths remain low in individuals receiving primary immunisation only, the booster programme will provide additional protection to those at highest risk of severe COVID-19 and help reduce infection 
rates across the population. ${ }^{10}$ Ongoing surveillance will be important for monitoring duration of protection offered by booster doses and any need for additional doses in the future.

\section{Acknowledgments}

We would like to thank Dorothy Blundell, Dr Caroline Sayer and the team at Haverstock Healthcare GP Federation, and the whole CONSESUS team at PHE including those within the Virus Reference Department at Colindale who assisted with the laboratory testing.

\section{Funding}

This surveillance was funded by Public Health England (PHE) (now UK Health Security Agency[UKHSA]). The CONSENSUS study/audit was approved by the PHE R\&D Research Ethics and Governance Group. No: NR0253

\section{Data availability}

Applications for relevant anonymised data should be submitted to the UK Health Security Agency Office for Data Release.

\section{Author Contributions}

$\mathrm{SL}, \mathrm{FB}, \mathrm{PM}, \mathrm{MR}, \mathrm{KB}, \mathrm{GA}$ conceived and designed the study; LW and EL supervised the laboratory testing; SS, CW, MO'B and FB co-ordinated the patient enrolment. HW performed the statistical analysis and GI, GA, SL, and KB wrote the manuscript. All authors read and approved the submission.

\section{Declaration of Interests}

MER reports that the Immunisation and Vaccine Preventable Diseases Division (UKHSA) has provided vaccine manufacturers with post-marketing surveillance reports on pneumococcal and meningococcal infection, which the companies are required to submit to the UK licensing authority in compliance with their risk management strategy. A cost-recovery charge is made for these reports. EL report that the UKHSA Vaccine Evaluation Unit does contract research on behalf of GlaxoSmithKline, Sanofi, and Pfizer, which is outside the submitted work. 
medRxiv preprint doi: https://doi.org/10.1101/2021.11.22.21266692; this version posted November 24, 2021. The copyright holder for this preprint (which was not certified by peer review) is the author/funder, who has granted medRxiv a license to display the preprint in perpetuity.

It is made available under a CC-BY 4.0 International license .

Table 1: Characteristics of CONSENSUS participants with samples after dose 2 of the primary vaccination and booster vaccine.

\begin{tabular}{|l|r|r|r|r|r|r|}
\hline vaccine & N & $\begin{array}{r}\text { dose 1-2 } \\
\text { schedule } \\
\text { median } \\
\text { (IQR) }\end{array}$ & $\begin{array}{r}\text { dose 2-3 } \\
\text { schedule } \\
\text { median (IQR) }\end{array}$ & $\begin{array}{r}\text { age } \\
\text { median } \\
\text { (IQR) }\end{array}$ & sex M (\%) & $\begin{array}{r}\text { ethnicity } \\
\text { non- } \\
\text { white (\%) }\end{array}$ \\
\hline all with samples after dose 2 & & & & & \\
\hline AZD1222 extended & 240 & $72(56-77)$ & & $73(55-71)$ & $109(45 \%)$ & $71(30 \%)$ \\
\hline BNT162b2 extended & 299 & $76(73-77)$ & & $76(75-80)$ & $42(48 \%)$ & $9(10 \%)$ \\
\hline BNT162b2 control & 87 & $21(21-21)$ & & & & \\
\hline all with samples after dose 3 & & & & & & \\
\hline AZD1222 extended & 50 & $56(51-70)$ & $186(182-190)$ & $69(66-71)$ & $20(40 \%)$ & $5(10 \%)$ \\
\hline BNT162b2 extended & 131 & $76(74-76)$ & $186(182-188)$ & $72(69-75)$ & $56(43 \%)$ & $10(8 \%)$ \\
\hline BNT162b2 control & 52 & $21(21-21)$ & $262(261-263)$ & $77(75-80)$ & $27(52 \%)$ & $4(8 \%)$ \\
\hline
\end{tabular}


medRxiv preprint doi: https://doi.org/10.1101/2021.11.22.21266692; this version posted November 24, 2021. The copyright holder for this preprint (which was not certified by peer review) is the author/funder, who has granted medRxiv a license to display the preprint in perpetuity.

It is made available under a CC-BY 4.0 International license .

Table 2: Geometric mean response and geometric mean ratio of response of CONSENSUS participants before and after dose 2 of the primary vaccination and booster vaccine for AZD1222 extended, BNT162 b2 control and BNT162b2 extended participants.

Geometric mean response was not calculated for categories with $<5$ people within them

\begin{tabular}{|c|c|c|c|c|c|c|}
\hline Vaccine & dose & $\begin{array}{l}\text { time } \\
\text { sampled } \\
\text { after } \\
\text { dose, } \\
\text { weeks }\end{array}$ & $\mathrm{n}$ & $\begin{array}{l}\text { geometric mean } \\
\text { responses }(95 \% \mathrm{Cl})\end{array}$ & $\begin{array}{l}\text { within-individual } \\
\text { geometric mean } \\
\text { ratio of response } \\
\text { relative to } 2-4 \\
\text { wks }(95 \% \mathrm{Cl})\end{array}$ & $\begin{array}{l}\text { within-individual } \\
\text { geometric mean } \\
\text { ratio of response } \\
\text { relative to } 0-3 \text { weeks } \\
\text { before dose }(95 \% \mathrm{Cl})\end{array}$ \\
\hline \multirow{10}{*}{$\begin{array}{l}\text { AZD1222 } \\
\text { extended }\end{array}$} & dose 1 & $<3$ pre $\mathrm{D} 2$ & 147 & $29(23-36)$ & & ref \\
\hline & \multirow{7}{*}{ dose 2} & 1 & 102 & $735(590-916)$ & $0.92(0.81-1.05)$ & $24.7(19.5-31.2)$ \\
\hline & & $2-4$ & 126 & $812(650-1015)$ & ref & $28.6(23-35.6)$ \\
\hline & & $5-8$ & 86 & $615(493-767)$ & $0.73(0.64-0.84)$ & \\
\hline & & $9-12$ & 61 & $487(364-652)$ & $0.53(0.46-0.62)$ & \\
\hline & & $13-17$ & 71 & $338(257-446)$ & $0.37(0.32-0.43)$ & \\
\hline & & $18-23$ & 74 & $258(194-343)$ & $0.31(0.27-0.36)$ & \\
\hline & & $24-29$ & 43 & $182(125-265)$ & $0.22(0.18-0.26)$ & \\
\hline & \multirow{2}{*}{ dose 3} & $<3$ pre D3 & 29 & $189(131-273)$ & & ref \\
\hline & & $2-4$ & 43 & $10799(8510-13704)$ & & $57.2(38.8-84.2)$ \\
\hline \multirow{5}{*}{$\begin{array}{l}\text { BNT162b2 } \\
\text { control }\end{array}$} & \multirow{3}{*}{ dose 2} & $2-4$ & 80 & $694(540-893)$ & ref & \\
\hline & & $18-23$ & 72 & $330(261-418)$ & $0.49(0.4-0.6)$ & \\
\hline & & $36-38$ & 56 & $208(150-289)$ & $0.32(0.25-0.39)$ & \\
\hline & \multirow{2}{*}{ dose 3} & $<3$ pre D3 & 38 & $233(162-336)$ & & ref \\
\hline & & $2-4$ & 47 & $18104(13911-23560)$ & & $76.3(58.1-100.1)$ \\
\hline \multirow{10}{*}{$\begin{array}{l}\text { BNT162b2 } \\
\text { extended }\end{array}$} & dose 1 & $<3$ pre $\mathrm{D} 2$ & 218 & $29(25-34)$ & & ref \\
\hline & \multirow{7}{*}{ dose 2} & 1 & 142 & $7442(6021$ - 9198) & $1.31(1.21-1.43)$ & $272.6(234.4-317)$ \\
\hline & & $2-4$ & 205 & $6621(5817-7536)$ & ref & $217.8(190.9-248.6)$ \\
\hline & & $5-8$ & 71 & $4930(4096-5934)$ & $0.75(0.67-0.84)$ & \\
\hline & & $9-12$ & 106 & $2698(2323-3135)$ & $0.43(0.39-0.47)$ & \\
\hline & & $13-17$ & 173 & 1770 (1545 - 2029) & $0.29(0.27-0.32)$ & \\
\hline & & $18-23$ & 43 & $1344(1081-1670)$ & $0.2(0.17-0.23)$ & \\
\hline & & $24-29$ & 126 & $942(797-1113)$ & $0.15(0.13-0.16)$ & \\
\hline & \multirow{2}{*}{ dose 3} & $<3$ & 91 & $854(697-1047)$ & & ref \\
\hline & & $2-4$ & 118 & $13980(11902-16421)$ & & $15.9(13.4-18.9)$ \\
\hline \multicolumn{7}{|c|}{ Past positives } \\
\hline \multirow{9}{*}{$\begin{array}{l}\text { AZD1222 } \\
\text { extended }\end{array}$} & dose 1 & $<3$ pre D2 & 39 & $8022(5655$ - 11379) & & ref \\
\hline & \multirow{7}{*}{ dose 2} & 1 & 25 & 9138 (5997 - 13924) & $1.19(0.94-1.51)$ & $1.1(0.9-1.3)$ \\
\hline & & $2-4$ & 33 & $7870(4547-13623)$ & ref & $1(0.9-1.2)$ \\
\hline & & $5-8$ & 22 & $7604(4997-11570)$ & $0.82(0.64-1.05)$ & \\
\hline & & $9-12$ & 12 & $6667(3549-12526)$ & $0.71(0.52-0.97)$ & \\
\hline & & $13-17$ & 19 & $4895(2949-8125)$ & $0.63(0.49-0.82)$ & \\
\hline & & $18-23$ & 15 & $6830(4021-11601)$ & $0.51(0.38-0.68)$ & \\
\hline & & $24-29$ & 5 & $2878(1367-6062)$ & $0.49(0.33-0.75)$ & \\
\hline & dose 3 & $<3$ pre D3 & 5 & $4583(2394-8772)$ & & ref \\
\hline
\end{tabular}


medRxiv preprint doi: https://doi.org/10.1101/2021.11.22.21266692; this version posted November 24, 2021. The copyright holder for this preprint (which was not certified by peer review) is the author/funder, who has granted medRxiv a license to display the preprint in perpetuity.

It is made available under a CC-BY 4.0 International license .

\begin{tabular}{|c|c|c|c|c|c|c|}
\hline & & $2-4$ & 6 & $45430(30374-67947)$ & & $9.9(6.4-15.4)$ \\
\hline \multirow{5}{*}{$\begin{array}{l}\text { BNT162b2 } \\
\text { control }\end{array}$} & \multirow{3}{*}{ dose 2} & $2-4$ & 7 & $17998(4379-73982)$ & & \\
\hline & & $19-21$ & 7 & $5317(934-30271)$ & & \\
\hline & & $36-38$ & 5 & $3357(277-40690)$ & & \\
\hline & \multirow{2}{*}{ dose 3} & $<3$ pre D3 & 3 & & & \\
\hline & & $2-4$ & 4 & & & \\
\hline \multirow{10}{*}{$\begin{array}{l}\text { BNT162b2 } \\
\text { extended }\end{array}$} & dose 1 & $<3$ pre $\mathrm{D} 2$ & 28 & $1797(768-4201)$ & & ref \\
\hline & dose 2 & 1 & 21 & $30920(20331-47025)$ & $1.53(1.31-1.79)$ & $18.6(9.7-35.9)$ \\
\hline & & $2-4$ & 25 & $24680(15900-38308)$ & ref & $12.6(6.8-23.2)$ \\
\hline & & $5-8$ & 7 & $11975(5327-26917)$ & $0.6(0.48-0.76)$ & \\
\hline & & $9-12$ & 14 & $8563(6121$ - 11979) & $0.48(0.41-0.57)$ & \\
\hline & & $13-17$ & 26 & $6221(4651-8321)$ & $0.33(0.28-0.38)$ & \\
\hline & & $18-23$ & 4 & & & \\
\hline & & $24-29$ & 20 & 4185 (2944 - 5949) & $0.2(0.17-0.23)$ & \\
\hline & \multirow{2}{*}{ dose 3} & $<3$ pre D3 & 10 & $4023(2215-7304)$ & & ref \\
\hline & & $2-4$ & 12 & $18604(12899-26832)$ & & $4.8(3.2-7.3)$ \\
\hline \multicolumn{3}{|c|}{$\begin{array}{l}\text { Convalescent sera, by weeks post } \\
\text { symptom onset }\end{array}$} & & & & \\
\hline \multirow{2}{*}{\multicolumn{2}{|c|}{$\begin{array}{l}\text { Unvaccinated, ages } \\
\text { 50-89 }\end{array}$}} & $3-7$ & 141 & $55.3(39.4,77.7)$ & & \\
\hline & & $8-12$ & 87 & $128.2(89.2,184.3)$ & & \\
\hline
\end{tabular}


medRxiv preprint doi: https://doi.org/10.1101/2021.11.22.21266692; this version posted November 24, 2021. The copyright holder for this preprint (which was not certified by peer review) is the author/funder, who has granted medRxiv a license to display the preprint in perpetuity.

It is made available under a CC-BY 4.0 International license .

Figure 1: Violin plots, $\mathrm{S}$ antibody responses $<3$ weeks before the $2^{\text {nd }}$ and booster dose of COVID-19 vaccine in previously uninfected individuals whose primary vaccination type was AZD1222-extended schedule, by interval between doses, BNT162b2-extended schedule, by interval between doses and BNT162b2-control schedule.

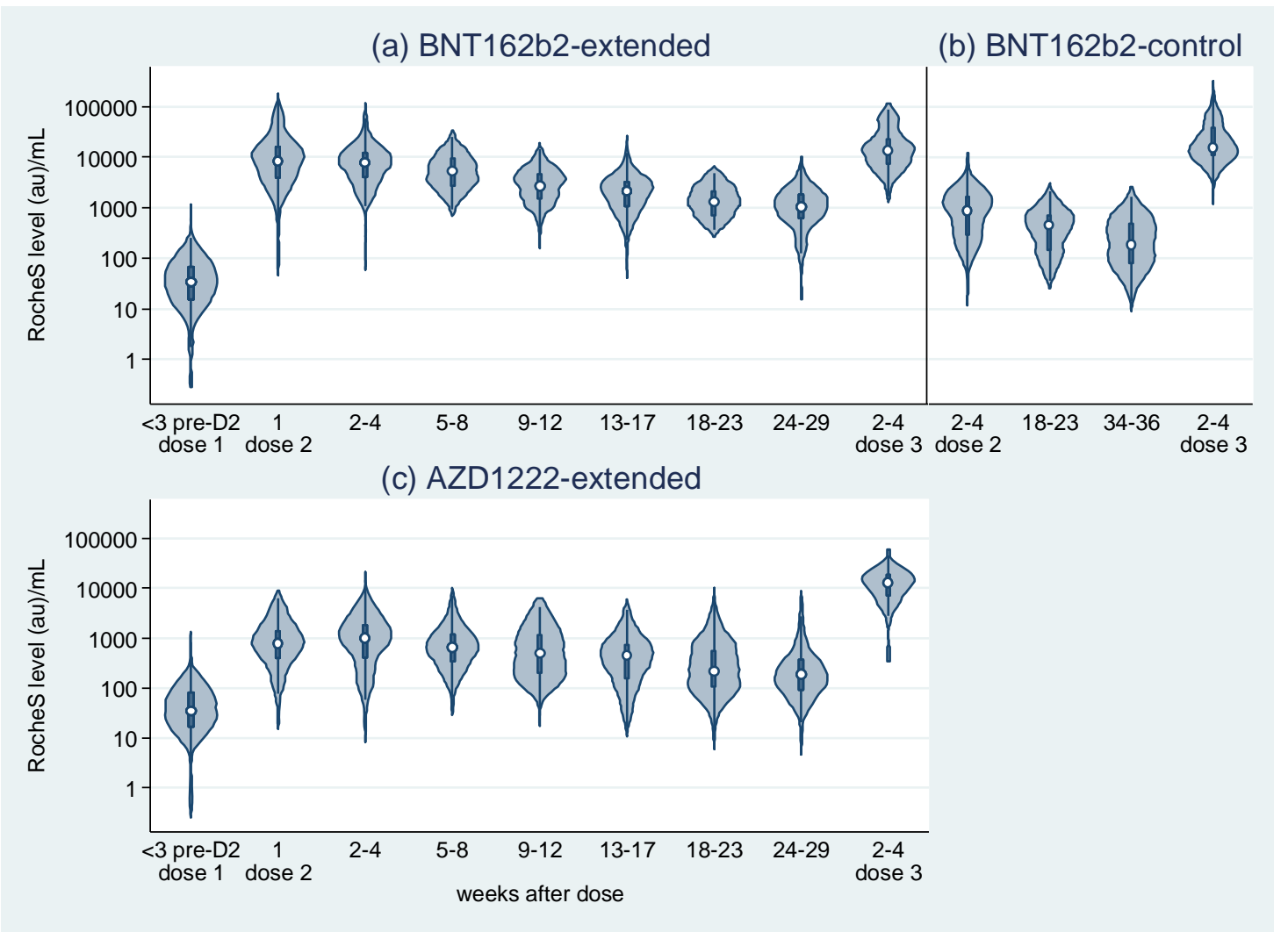




\section{References}

1. Amirthalingam G, Lopez Bernal J, Andrews N, et al. Higher serological responses and increased vaccine effectiveness demonstrate the value of extended vaccine schedules in combatting COVID-19 in England. Medrxiv 2021.

2. Andrews N, Stowe J, Kirsebom F, Gower C, Ramsay ME, Lopez Bernal J. Effectiveness of BNT162b2 (Comirnaty, Pfizer-BioNTech) COVID-19 booster vaccine against covid-19 related symptoms in England: test negative case-control study. Medrxiv 2021.

3. Twohig KA, Nyberg T, Zaidi A, et al. Hospital admission and emergency care attendance risk for SARS-CoV-2 delta (B.1.617.2) compared with alpha (B.1.1.7) variants of concern: a cohort study. Lancet Infect Dis 2021.

4. Lopez Bernal J, Andrews N, Gower C, et al. Effectiveness of Covid-19 Vaccines against the B.1.617.2 (Delta) Variant. N Engl J Med 2021; 385(7): 585-94.

5. The Joint Committee on Vaccination and Immunisation. JCVI issues advice on COVID-19 booster vaccines for those aged 40 to 49 and second doses for 16 to 17 year olds. 2021.

6. Chemaitelly H, Tang P, Hasan MR, et al. Waning of BNT162b2 Vaccine Protection against SARS-CoV-2 Infection in Qatar. N Engl J Med 2021.

7. UK Health Security Agency. Weekly national Influenza and COVID-19 surveillance report:

Week 27 report (up to week 26 data), 2021.

8. Subbarao S, Warrener LA, Hoschler K, et al. Robust antibody responses in 70-80-year-olds 3 weeks after the first or second doses of Pfizer/BioNTech COVID-19 vaccine, United Kingdom, January to February 2021. Euro Surveill 2021; 26(12).

9. Barda N, Dagan N, Cohen C, et al. Effectiveness of a third dose of the BNT162b2 mRNA COVID-19 vaccine for preventing severe outcomes in Israel: an observational study. The Lancet 2021; Online First.

10. Gardener BJ, Kilpatrick AM. Third doses of COVID-19 vaccines reduce infection and transmission of SARS-CoV-2 and could prevent future surges in some populations

Medrxiv 2021. 